\title{
In-hospital cardiac arrest in piedmont (ITALY): epidemiology and outcomes
}

\author{
A Mira', G Radeschi², G Berta ${ }^{1}$, F Rubulotta ${ }^{3^{*}}$ \\ From ESICM LIVES 2015 \\ Berlin, Germany. 3-7 October 2015
}

\section{Introduction}

Hospitals situated in the Piedmont Region (Italy) have systematically collected data on in-hospital cardiac arrest (IHCA) since 2012. This activity has been established as part of the Regional Guidelines for the continuous quality improvement of the in-hospital emergency. Those guidelines and improvement strategies are coordinated by the Scientific Committee of the Regional Board and transferred to the Monitoring Center (CdM) that takes care of the data quality control, and the preparation of an annual report.

\section{Objectives}

The aim of this study is to describe the epidemiology, nature, incidence and clinical outcomes of IHCA over twelve consecutive months (January $1^{\text {st }}$ to December $31^{\text {st }}$, 2013) in Piedmont Region (35 Hospitals, 618 consecutive patients). Our database analysis could be valuable for comparison/benchmarking with national and international data/standards ${ }^{(1)}$.

\section{Methods}

Systematic data collection was performed using the Italian Cardiac Arrest Register (RIAC). This is an electronic online database created by the Italian Resuscitation Council (IRC). Within the database, the 35 Piedmont hospitals form a cluster. The records were collected using the Utstein standard. Ethics approval was obtained by the local ethics review board and all patient' information were collected anonymously and analyzed by the CdM.

\section{Results}

The CdM reviewed over 12 months a total of 618 patients in 25 centers. The patients' characteristics are shown in Table 1 and the outcomes in Table 2. The incidence of

${ }^{3}$ Imperial College London, London, United Kingdom

Full list of author information is available at the end of the article
IHCA was 1.7/1000 admissions/year. The presenting rhythm was shockable in $23,1 \%$ and ROSC was obtained in 228 cases $(41,1 \%)$. The survivors at hospital discharge were 79 (14,2\% of the CPR started), 73 of those $(92,4 \%)$ with good neurological outcomes (CPC 1-2). Follow-up at 6 months showed 60 patients $(10,8 \%)$ are still alive.

\section{Conclusions}

35 hospitals collaborated in Piedmont collecting data regarding IHCA. The incidence of IHCA is comparable with one of the most recent international studies ${ }^{(1)}$. Major limitation of this study is the fact that we recorded only the events involving the MET. Therefore, we cannot exclude other events occurred without involving the medical emergency team. The percentage of shockable rhythms was slightly lower than the average reported in the literature. We believe that epidemiological data and outcome data recorded for IHCA in Piedmont could be compared and benchmark with other centers at a national and international level. Therefore, we are confident that this study will help improving the treatment of cardiac arrest and the organization of the emergency systems.

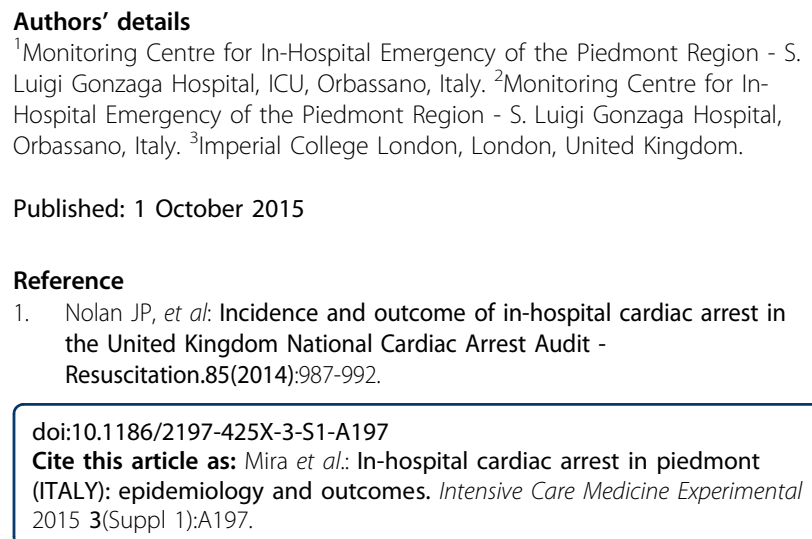

Published: 1 October 2015

Reference

1. Nolan JP, et al: Incidence and outcome of in-hospital cardiac arrest in the United Kingdom National Cardiac Arrest Audit -

Resuscitation.85(2014):987-992.

doi:10.1186/2197-425X-3-S1-A197

Cite this article as: Mira et al.: In-hospital cardiac arrest in piedmont (ITALY): epidemiology and outcomes. Intensive Care Medicine Experimental 2015 3(Suppl 1):A197.

\section{SpringerOpen $^{\circ}$}

(c) 2015 Rubulotta et al.; This is an Open Access article distributed under the terms of the Creative Commons Attribution License (http://creativecommons.org/licenses/by/4.0), which permits unrestricted use, distribution, and reproduction in any medium, provided the original work is properly cited. 